Journal of Algebra Combinatorics Discrete Structures and Applications

\title{
Fourier matrices of small rank
}

\section{Gurmail Singh}

Abstract: Modular data is an important topic of study in rational conformal field theory. Cuntz, using a computer, classified the Fourier matrices associated to modular data with rational entries up to rank 12, see [3]. Here we use the properties of $C$-algebras arising from Fourier matrices to classify complex Fourier matrices under certain conditions up to rank 5. Also, we establish some results that are helpful in recognizing $C$-algebras that not arising from Fourier matrices by just looking at the first row of their character tables.

2010 MSC: 05E30, 05E99, 81R05

Keywords: Fourier matrices, Modular data, Fusion rings, $C$-algebras

\section{Introduction}

Fourier matrices are a fundamental ingredient of modular data. Modular data is a basic component of rational conformal field theory, see [5]. Further, rational conformal field theory has important applications in physics, see [4] and [8]. In particular, it has nice applications to string theory, statistical mechanics, and condensed matter physics, see [10] and [13]. Modular data give rise to fusion rings, $C$-algebras and $C^{*}$-algebras, see [3] and [11]. These rings and algebras are interesting topics of study in their own right.

A unitary and symmetric matrix whose first column has positive real entries is called a Fourier matrix if its columns under entrywise multiplication produce integral structure constants. The set of columns of a Fourier matrix under entrywise multiplication and usual addition generate a fusion algebra, see [3]. But a two-step rescaling on Fourier matrices gives rise to self-dual $C$-algebras. Cuntz, using a computer, classified the Fourier matrices with rational entries up to rank 12, see [3]. But rational Fourier matrices do not include some other important matrices, see sections 4,5 and 6 . Here we use $C$-algebra perspective to classify the complex Fourier matrices up to rank 5 under certain conditions. Also, we establish some results that are helpful in recognizing the $C$-algebras that are not arising from Fourier matrices by mere looking at the first row of their character tables.

Gurmail Singh; Department of Mathematics and Statistics, University of Regina, Canada, S4S 0A2 (email: gurmail.singh@uregina.ca). 
In Section 2, we collect the definitions and introduce a two-step rescaling of Fourier matrices. In Section 3, we summarize the results that are useful to recognize the $C$-algebras that are not arising from Fourier matrices. In Section 4, we classify Fourier matrices of rank 2 and 3. In sections 5 and 6 , we classify non-homogeneous Fourier matrices of rank 4 and rank 5, respectively. For the classification of homogenous Fourier matrices see [11, Theorem 13].

\section{2. $\quad C$-algebras arising from Fourier matrices}

A scaling of the rows of a Fourier matrix gives the basis of a Fusion algebra that contains the identity element. But a two-step rescaling of a Fourier matrix gives the standard basis of $C$-algebra.

Definition 2.1. Let $A$ be a finite dimensional and commutative algebra over $\mathbb{C}$ with distinguished basis $\mathbf{B}=\left\{b_{0}:=1_{A}, b_{1}, \ldots, b_{r-1}\right\}$, and an $\mathbb{R}$-linear and $\mathbb{C}$-conjugate linear involution $*: A \rightarrow A$. Let $\delta: A \rightarrow \mathbb{C}$ be an algebra homomorphism. Then the triple $(A, \mathbf{B}, \delta)$ is called a $C$-algebra if it satisfies the following properties:

1. for all $b_{i} \in \mathbf{B},\left(b_{i}\right)^{*}=b_{i^{*}} \in \mathbf{B}$,

2. for all $b_{i}, b_{j} \in \mathbf{B}$, we have $b_{i} b_{j}=\sum_{b_{k} \in \mathbf{B}} \lambda_{i j k} b_{k}$, for some $\lambda_{i j k} \in \mathbb{R}$,

3. for all $b_{i}, b_{j} \in \mathbf{B}, \lambda_{i j 0} \neq 0 \Longleftrightarrow j=i^{*}$,

4. for all $b_{i} \in \mathbf{B}, \lambda_{i i^{*} 0}=\lambda_{i^{*} i 0}>0$.

5. for all $b_{i} \in \mathbf{B}, \delta\left(b_{i}\right)=\delta\left(b_{i^{*}}\right)>0$.

The algebra homomorphism $\delta$ is called a degree map, and the values $\delta\left(b_{i}\right)$, for all $b_{i} \in \mathbf{B}$, are called the degrees of $A$. For $i \neq 0, \delta\left(b_{i}\right)$ is called a nontrivial degree. If $\delta\left(b_{i}\right)=\lambda_{i i^{*} 0}$, for all $b_{i} \in \mathbf{B}$, we say that $\mathbf{B}$ is a standard basis. The order of a $C$-algebra is denfined as $\delta\left(\mathbf{B}^{+}\right):=\sum_{i=0}^{r-1} \delta\left(b_{i}\right)$. A $C$-algebra is called symmetric if $b_{i^{*}}=b_{i}$, for all $i$. A $C$-algebra with rational structure constants is called a rational $C$-algebra. The readers interested in $C$-algebras are directed to [1], [2] and [7].

To keep the generality, in the following definition of a Fourier matrix we assume the structure constants to be integers instead of nonnegative integers, see [3, Definition 2.2].

Definition 2.2. Let $r \in \mathbb{Z}^{+}$and $I$ an $r \times r$ identity matrix. Then $S$ is called a Fourier matrix if

1. $S$ is a unitary and symmetric matrix, that is, $S \bar{S}^{T}=I, S=S^{T}$,

2. $S_{i 0}>0$, for $0 \leq i \leq r-1$, where $S$ is indexed by $\{0,1,2, \ldots, r-1\}$,

3. $N_{i j k}=\sum_{l} S_{l i} S_{l j} \bar{S}_{l k} S_{l 0}^{-1} \in \mathbb{Z}$, for all $0 \leq i, j, k \leq r-1$.

Let $S$ be a Fourier matrix. Let $s=\left[s_{i j}\right]$ be a matrix with entries $s_{i j}=S_{i j} / S_{i 0}$, for all $i, j$, we call it an $s$-matrix associated to $S$ (briefly, $s$-matrix). Since $S$ is a unitary matrix, $s \bar{s}^{T}=\operatorname{diag}\left(d_{0}, d_{1}, \ldots, d_{r-1}\right)$ is a diagonal matrix, where $d_{i}=\sum_{j} s_{i j} \bar{s}_{i j}$. The numbers $d_{i}$ are called norms of $s$-matrix. The relation $s_{i j}=S_{i j} / S_{i 0}$ implies the structure constants $N_{i j k}=\sum_{l} s_{l i} s_{l j} s_{l k} d_{l}^{-1}$, for all $i, j, k$. Since the structure constants $N_{i j k}$ are integers, the numbers $s_{i j}$ are algebraic integers, see [3, Section 3]. Therefore, if $S$ has only rational entries then entries of $s$-matrix are rational integers, and such $s$-matrices are known as integral Fourier matrices, see [3, Definition 3.1]. Cuntz classified the integral Fourier matrices up to rank 12 by using a computer, see [3]. In this paper, we consider the broader class of $s$-matrices that have algebraic integer entries.

There is an interesting row-and-column operation (two-step rescaling) procedure that can be applied to a Fourier matrix $S$ that results in the first eigenmatrix, the character table, of a self-dual $C$-algebra. The steps of the procedure are reversed to obtain the Fourier matrix $S$ from the first eigenmatrix. The 
explanation of the procedure is as follows. Let $S=\left[S_{i j}\right]$ be a Fourier matrix indexed with $\{0,1, \ldots, r-1\}$. We divide each row of $S$ with its first entry and obtain the $s$-matrix. The multiplication of each column of the $s$-matrix with its first entry gives the $P$-matrix associated to $S$ (briefly, $P$-matrix), the first eigenmatrix of a self-dual $C$-algebra. That is, $s_{i j}=S_{i j} S_{i 0}^{-1}$ and $p_{i j}=s_{i j} s_{0 j}$, for all $i, j$, where $p_{i j}$ denotes the $(i, j)$-entry of the $P$-matrix. Conversely, to obtain the $s$-matrix from a $P$-matrix, divide each column of the $P$-matrix with the squareroot of its first entry. Further, the Fourier matrix $S$ is obtained from the $s$-matrix by dividing the $i$ th row of $s$-matrix by $\sqrt{d_{i}}$, where $d_{i}=\sum_{j}\left|s_{i j}\right|^{2}$. That is, $s_{i j}=p_{i j} / \sqrt{p_{0 j}}$, and $S_{i j}=s_{i j} / \sqrt{d_{i}}$, for all $i, j$. Since the entries of an $s$-matrix are algebraic integers, the entries of a $P$-matrix are also algebraic integers.

Remark 2.3. Throughout this paper, unless mentioned explicitly, the sets of columns of a $P$-matrix and an $s$-matrix are denoted by $\mathbf{B}=\left\{b_{0}, b_{1}, \ldots, b_{r-1}\right\}$ and $\tilde{\mathbf{B}}=\left\{\tilde{b}_{0}, \tilde{b}_{1}, \ldots, \tilde{b}_{r-1}\right\}$, respectively. The structure constants generated by the columns, with entrywise multiplication, of a $P$-matrix and an $s$-matrix are denoted by $\lambda_{i j k}$ and $N_{i j k}$, respectively. $M^{T}$ denotes the transpose of a matrix $M$.

Let $S$ be a Fourier matrix and $A:=\mathbb{C B}$, a $\mathbb{C}$-span of B. Define a map $*: A \longrightarrow A$ by $\left(\sum_{j} a_{j} b_{j}\right)^{*}=$ $\sum_{j} \bar{a}_{j} b_{j^{*}}=\sum_{j} \bar{a}_{j}\left[\bar{p}_{0 j}, \bar{p}_{1 j}, \ldots, \bar{p}_{r-1, j}\right]^{T}$. This map $*$ is an involution on $A$, and the map $\delta: A \longrightarrow \mathbb{C}$ defined as $\delta\left(\sum_{j} a_{j} \tilde{b}_{j}\right)=\sum_{j} \bar{a}_{j} s_{0 j}$, that is, $\delta\left(b_{i}\right)=\delta\left(s_{0 i} \tilde{b}_{i}\right)=s_{0 i}^{2}$ for all $i$, is a positive degree map of $A$. Since $b_{i}=s_{0 i} \tilde{b}_{i}$, the structure constants generated by the basis $\mathbf{B}$ are given by $\lambda_{i j k}=N_{i j k} s_{0 i} s_{0 j} s_{0 k}^{-1}$, for all $i, j, k . S$ is a unitary matrix, therefore, $N_{i j 0}=\sum_{l} S_{l i} S_{l j} \bar{S}_{l 0} S_{l 0}^{-1} \neq 0 \Longleftrightarrow j=i^{*}$ and $N_{i i^{*} 0}=1>0$, for all $i, j$. Thus $\lambda_{i j 0} \neq 0 \Longleftrightarrow j=i^{*}$ and $\lambda_{i i^{*} 0}>0$, for all $i, j$. Therefore, the vector space $A:=\mathbb{C B}$ is a $C$-algebra of order $d_{0}, \mathbf{B}$ is the standard basis of $A$, and $P$-matrix is the first eigenmatrix of $A$, see [11, Theorem 4], and we say $(A, \mathbf{B}, \delta)$ is a $C$-algebra arising from a Fourier matrix $S$. Note that, entries of the first eigenmatrix $P$ are the entries of the character table $A$. Thus at some places we consider the $P$-matrix of $A$ as the character table of $A$ and the $i$ th row of $P$-matrix as the $i$ th irreducible character of $A$.

Let $(A, \mathbf{B}, \delta)$ be a $C$-algebra arising from a Fourier matrix $S$. Since $S$ is a symmetric matrix, $A$ is a self-dual $C$-algebra and $d_{0}=d_{j} \delta\left(b_{j}\right)$, for all $j$. The entries of an $s$-matrix and the associated $P$-matrix are algebraic integers. Therefore, if $A$ has rational degrees then both the degrees and norms are rational integers and both divide the order of $A$, see [11, Proposition 5]. Note that, a $C$-algebra arising from a Fourier matrix $S$ is a symmetric $C$-algebra if and only if $S$ is a real matrix. A $C$-algebra that has at least two different nontrivial degrees is called a non-homogeneous $C$-algebra and we call the associated Fourier matrix (s-matrix) a non-homogeneous Fourier matrix (non-homogeneous s-matrix, respectively).

Every self-dual $C$-algebra not necessarily have rational degrees. For example, a self-dual $C$-algebra of rank 2 with basis $\{1, x\}$, and the structure constants given by the equation $x^{2}=1+x$ does not have rational degrees. We remark that this $C$-algebra does not arise from a Fourier matrix. But a rational $C$-algebra arising from a Fourier matrix has integral degrees, see [11, Proposition 5].

Lemma 2.4. Let $(A, \mathbf{B}, \delta)$ be a $C$-algebra arising from a Fourier matrix $S$ of rank $r$.

1. If $A$ has rational order then the order of $A$ is an integer.

2. If $A$ has nonnegative structure constants then degrees of $A$ are greater or equal to 1 .

3. If $A$ has rational order and all the degrees of $A$ different from 1 are all equal then the degrees of $A$ are integers. (Note: the algebra A need not be homogeneous.)

Proof. (i). Since degrees of $A$ are algebraic integers and order of $A$ is the sum of degrees of $A$, the order of $A$ is a rational integer.

(ii). For any $i, \delta\left(b_{i}\right)$ is the first entry of column vector $\left(\tilde{b}_{i}\right)^{2}$ and $N_{i i 0}=1$. Note that, $N_{i j k} \geq 0$ for all $i, j, k$, because $A$ has nonnegative structure constants. Therefore, $\delta\left(b_{i}\right)=1+m$, where $m$ is a nonnegative algebraic integer.

(iii). Let all the degrees of $A$ different from 1 be a positive real number $t$. Therefore, $d_{0}=m+n t$, where $m$ is the number of degrees equal to 1 and $n$ is the number of degrees equal to $t$. Since the order $d_{0}$ is an integer, $t$ is an integer. 


\section{Recognition of $C$-algebras arising from Fourier matrices}

The following results are useful for recognizing $C$-algebras that are not arising from Fourier matrices by mere looking at the degrees of $C$-algebras, that is, the first row of the character tables. All the character tables of the association schemes used here are produced by Hanaki and Miyamoto, see [6].

Lemma 3.1. Let $(A, \mathbf{B}, \delta)$ be a $C$-algebra arising from a Fourier matrix $S$ with nonnegative structure constants. Let $L(\mathbf{B})=\{b \in \mathbf{B}: \delta(b)=1\}$. Then $L(\mathbf{B})$ is an abelian group.

Proof. Since $\delta\left(b_{0}\right)=1, b_{0} \in L$. Let $b_{i}, b_{j} \in L(\mathbf{B})$. Therefore, $b_{i}=\tilde{b}_{i}$ and $b_{j}=\tilde{b}_{j}$. Thus $b_{i} b_{j}=\tilde{b}_{i} \tilde{b}_{j}=$ $\sum_{k} N_{i j k} \tilde{b}_{k}$ implies $1=\delta\left(b_{i} b_{j}\right)=\sum_{k} N_{i j k} \delta\left(\tilde{b}_{k}\right)$. By Lemma 2.4, $\delta\left(b_{l}\right) \geq 1$, thus $\delta\left(\tilde{b}_{l}\right)=\sqrt{\delta\left(b_{l}\right)} \geq 1$, for all $l$. Therefore, $b_{i} b_{j}=\tilde{b}_{k}$, for some $b_{k} \in \mathbf{B}$. Also, for all $b_{i} \in L(\mathbf{B}), b_{i} b_{i^{*}}=b_{0}+\sum_{j} \lambda_{i i^{*} j} b_{j}$ and $\lambda_{i i^{*} j} \geq 0$ imply $b_{i} b_{i^{*}}=b_{0}$, that is, $b_{i}^{-1}=b_{i^{*}} \in L(\mathbf{B})$. Hence $L(\mathbf{B})$ is an abelian group.

Proposition 3.2. Let $(A, \mathbf{B}, \delta)$ be a $C$-algebra arising from a Fourier matrix $S$ with nonnegative structure constants. Let $S$ be a real Fourier matrix.

1. Let the order of $A$ be a rational number. If all the degrees of $A$ different from 1 are equal to $t$ then $t$ might be a power of 2 .

2. If rank of $A$ is an even integer then $A$ cannot have only one degree different from 1.

3. Let the order of $A$ be a rational number. If the rank of $A$ is greater than 3 then $A$ cannot have only one degree greater or equal to $r$ and all other degrees equal to 1.

Proof. (i). Since $S$ is a real Fourier matrix, by Lemma 3.1, the elements of B with degree 1 form an elementary abelian group. Thus the number of elements of $\mathbf{B}$ with degree 1 is a power of 2 . By Lemma $2.4, t$ is an integer. The result follows from the fact that $t$ divides the order of the algebra, see [11, Proposition 5 (ii)].

(ii). If rank of $A$ is 2 then both degrees are equal to 1 , see Section 4. By Lemma 3.1, the set of elements of $\mathbf{B}$ with degree 1 form an elementary abelian group. Therefore, the order of the group is a power of 2 , say $2^{m}$, where $m$ is a nonnegative integer. Since $A$ has only one degree different from 1 , $2^{m}=r-1$, a contradiction to the fact that $r$ is an even integer.

(iii). Suppose $A$ has only one degree $k$ that is greater or equal to $r$ and the remaining degrees are equal to 1 . Without loss of generality, let the first row of the character table be $[1,1, \ldots, 1, k]$, where $k \geq r$. Since $\delta\left(b_{0}\right)=\ldots=\delta\left(b_{r-2}\right), d_{0}=\ldots=d_{r-2}$. The structure constants are nonnegative, therefore, $\left|p_{i j}\right| \leq p_{0 j}$ for all $i, j$, see [12, Proposition 4.1]. Therefore, the only possible entries of row $2, \ldots$, row $r-1$ of $P$-matrix are $[1,1, \ldots, 1,-k]$, which is not possible as $P$ nonsingular.

The adjacency algebras of the association schemes as12(9), as14(4), as16(10), as16(20), as16(21) and as16(62) have the degree patterns that violate the above result, see [6]. Therefore, they are not arising from Fourier matrices. The character table of the association scheme as4(2) [6] is an example where part (iii) of the above proposition fails for the rank 3 . The above proposition also helps to sieve out a lot of $C$-algebras even if their self-duality is not known.

The next proposition helps to recognize the $C$-algebras not arising from Fourier matrices.

Proposition 3.3. Let $(A, \mathbf{B}, \delta)$ be a $C$-algebra arising from a Fourier matrix $S$ of rational order. Let the number of $i$ 's such that $\delta\left(b_{i}\right)=1$ be $t$ and the remaining $r-t$ degrees are equal to $k$. Then the possible values of $k$ are the divisors of $t$.

Proof. By Lemma 2.4, the order and degrees of $A$ are integers. Since entries of $s$-matrix are algebraic integers, all the norms are also integer, that is, $d_{0} k^{-1} \in \mathbb{Z}$. Therefore, $d_{0}=t+\left(d_{0}-t\right) k$ implies $t k^{-1} \in \mathbb{Z}$. Hence $k$ is in the subset of the divisors of $t$. 
The above proposition illustrates that the adjacency algebras of the association schemes as9(3), as9(8), as10(6), as16(20), as16(21) and as16(62) [6] are not arising from Fourier matrices.

In the next proposition we examine the possible number of occurrences of a degree if it is one of the degrees and satisfy a certain criteria.

Proposition 3.4. Let $(A, \mathbf{B}, \delta)$ be a $C$-algebra arising from a Fourier matrix $S$ with integral degrees.

1. If for a given $j, \delta\left(b_{j}\right)(\neq 1)$ is a smallest nontrivial degree that divides all the nontrivial degrees $\delta\left(b_{l}\right)(\neq 1)$ then the number of degrees equal to 1 is a multiple of $\delta\left(b_{j}\right)$.

2. Let $\delta\left(b_{t}\right)$ be a degree divisible by all the smaller degrees and divides all the bigger degrees. Let $\delta\left(b_{s}\right)$ be the largest degree among all the degrees strictly less than $\delta\left(b_{t}\right)$. Let the sum of the degrees less than $\delta\left(b_{s}\right)$ be $\beta_{1} \delta\left(b_{s}\right)$ and the number of degrees equal to $\delta\left(b_{s}\right)$ be $\beta_{2}$. Then $\beta_{1} \delta\left(b_{s}\right)+\beta_{2} \delta\left(b_{s}\right)$ is divisible by $\delta\left(b_{s}\right)$. (Note: $\beta_{1} \delta\left(b_{s}\right)$ is not equal to zero only if $\delta\left(b_{s}\right)>1$.)

3. Suppose $A$ has nonnegative structure constants. If for all $i<j, \delta\left(b_{i}\right)$ divides $\delta\left(b_{j}\right)$ then $A$ has integral structure constants.

Proof. (i). Since degrees of $A$ are integers, the norms are integers. Thus, $d_{0} \delta\left(b_{j}\right)^{-1} \in \mathbb{Z}$. Therefore,

$$
\left(1+\sum_{i=1}^{r-1} \delta\left(b_{i}\right)\right) \delta\left(b_{j}\right)^{-1}=\left(\sum_{\delta\left(b_{i}\right)=1} \delta\left(b_{i}\right)+\sum_{\delta\left(b_{i}\right) \geq \delta\left(b_{j}\right)} \delta\left(b_{i}\right)\right) \delta\left(b_{j}\right)^{-1}=\left(\sum_{\delta\left(b_{i}\right)=1} \delta\left(b_{i}\right)\right) \delta\left(b_{j}\right)^{-1}+\alpha \in \mathbb{Z}
$$

where $\alpha \in \mathbb{Z}$. Hence the number of degrees equal to 1 are multiple of $\delta\left(b_{j}\right)$.

(ii). The degrees and norms of $A$ are integers. Therefore,

$$
\begin{aligned}
d_{0} \delta\left(b_{t}\right)^{-1} & =\left(\sum_{i=0}^{r-1} \delta\left(b_{i}\right)\right) \delta\left(b_{t}\right)^{-1}=\left(\sum_{\delta\left(b_{i}\right)<\delta\left(b_{s}\right)} \delta\left(b_{i}\right)+\sum_{\delta\left(b_{i}\right)=\delta\left(b_{s}\right)} \delta\left(b_{i}\right)+\sum_{\delta\left(b_{i}\right)>\delta\left(b_{s}\right)} \delta\left(b_{i}\right)\right) \delta\left(b_{t}\right)^{-1} \\
& =\left(\beta_{1} \delta\left(b_{s}\right)+\sum_{\delta\left(b_{i}\right)=\delta\left(b_{s}\right)} \delta\left(b_{i}\right)\right) \delta\left(b_{t}\right)^{-1}+\gamma \in \mathbb{Z}
\end{aligned}
$$

where $\gamma \in \mathbb{Z}$. Thus $\beta_{1} \delta\left(b_{s}\right)+\beta_{2} \delta\left(b_{s}\right)$ is divisible by $\delta\left(b_{t}\right)$.

(iii). Since $\lambda_{i j k}$ are nonnegative, $N_{i j k}$ are nonnegative, because $\lambda_{i j k}=N_{i j k} s_{0 i} s_{0 j} s_{0 k}^{-1}$, for all $i, j, k$. Let $\tilde{b_{i}} \tilde{b_{j}}=\sum_{k} N_{i j k} \tilde{b_{k}}$. On comparing the first entry of both sides, we conclude that $\tilde{b_{k}}$ cannot occur with nonzero coefficient whenever $s_{0 k}>s_{0 i} s_{0 j}$, that is, $\sqrt{\delta\left(b_{k}\right)}>\sqrt{\delta\left(b_{i}\right) \delta\left(b_{j}\right)}$ implies $N_{i j k}=0$. Hence the assertion follows from the relation between $\lambda_{i j k}$ and $N_{i j k}$.

For example, the adjacency algebras of association schemes as7(2), as8(5), as8(6), as9(8), as9(9) and adjacency algebras of homogenous schemes have the degree patterns that violate the above proposition, see [6]. Therefore, they are not arising from Fourier matrices.

Lemma 3.5. Let $(A, \mathbf{B}, \delta)$ be a $C$-algebra arising from a rational Fourier matrix $S$ of odd rank and odd order. Let the odd degree among all the degrees of $A$ be maximum. Then the rank of $A$ must be at least 11.

Proof. Let $d_{0}=\delta\left(b_{i}\right) a_{i}$. By [3, Lemma 3.7], $d_{0}$ is an odd square, thus $a_{i}$ is a square. Let $\delta\left(b_{1}\right)$ be an odd integer and $\delta\left(b_{1}\right) \geq \delta\left(b_{i}\right)$ for each $i$. Therefore, $d_{0} \geq 9 \delta\left(b_{1}\right)$, and $d_{0} \leq 1+(r-1) \delta\left(b_{1}\right)$. Thus $9 \delta\left(b_{1}\right) \leq 1+(r-1) \delta\left(b_{1}\right)$ implies $\delta\left(b_{1}\right)(9-(r-1)) \leq 1$ implies $\delta\left(b_{1}\right) \in \mathbb{Z}^{+}$only if $r-1 \geq 9$. 


\section{Fourier matrices of rank 2 and 3}

In this section we classify Fourier matrices of rank 2 and 3 . In fact, we find the $P$-matrices of $C$-algebras arising from Fourier matrices of rank 2 and 3. But the associated Fourier matrix $S$ can be recovered easily from the $P$-matrix as described in Section 2.

Since the row sum of a character table is zero, the character table of a $C$-algebra of rank 2 with standard basis $\mathbf{B}=\left\{b_{0}, b_{i}\right\}$ is given by $P=\left[\begin{array}{cc}1 & n \\ 1 & -1\end{array}\right]$, and the structure constants are given by $b_{1}^{2}=$ $n b_{0}+(n-1) b_{1}$. Therefore, the structure constant $N_{111}$ is integer only for $n=1$, and the associated Fourier matrix $S=\frac{1}{\sqrt{2}}\left[\begin{array}{cc}1 & n \\ 1 & -1\end{array}\right]$.

Let $P$ be the character table for a symmetric $C$-algebra arising from a Fourier matrix of rank 3 with standard basis $\mathbf{B}=\left\{b_{0}, b_{1}, b_{2}\right\}$. Let $b_{1} b_{2}=u b_{1}+v b_{2}$. Then

$$
P=\left[\begin{array}{lll}
1 & k_{1} & k_{2} \\
1 & \phi_{1} & \phi_{2} \\
1 & \psi_{1} & \psi_{2}
\end{array}\right]
$$

where $\phi_{1}=\left(v-u-1+\sqrt{(u-v-1)^{2}+4 u}\right) / 2, \quad \phi_{2}=\left(u-v-1-\sqrt{(u-v-1)^{2}+4 u}\right) / 2$, and $\psi_{1}=\left(v-u-1-\sqrt{(u-v-1)^{2}+4 u}\right) / 2, \quad \psi_{2}=\left(u-v-1+\sqrt{(u-v-1)^{2}+4 u}\right) / 2$. Therefore, $d_{0}=1+k_{1}+k_{2}, d_{1}=1+\frac{\left|\phi_{1}\right|^{2}}{k_{1}}+\frac{\left|\phi_{2}\right|^{2}}{k_{2}}, d_{2}=1+\frac{\left|\psi_{1}\right|^{2}}{k_{1}}+\frac{\left|\psi_{2}\right|^{2}}{k_{2}}$.

Lemma 4.1. There is no symmetric homogenous $C$-algebra of rank 3 arising from a Fourier matrix $S$.

Proof. Suppose $(A, \mathbf{B}, \delta)$ is a symmetric homogenous $C$-algebra arising from a Fourier matrix. Since $S^{T}=S, \phi_{2} k=\psi_{1} l$ implies $u=v$. The structure constant $N_{210}=0$ implies $k=2 u$. Thus $\phi_{1}=$ $(-1+\sqrt{1+2 k}) / 2$ and $\phi_{2}=(-1-\sqrt{1+2 k}) / 2$. A homogenous $C$-algebra arising from a Fourier matrix has all degrees equal to 1 , see [11, Proposition 12]. But the structure constants

$$
N_{112}=\frac{1}{(2 k+1) \sqrt{k}}\left[k^{2}+\frac{k}{2}\right] \text { and } N_{222}=\frac{1}{(2 k+1) \sqrt{k}}\left[k^{2}-1-\frac{3}{2} k\right]
$$

are not integers for $k=1$, a contradiction.

Theorem 4.2. Let $(A, \mathbf{B}, \delta)$ be a symmetric non-homogeneous $C$-algebra of rank 3 arising from a Fourier matrix $S$ with integral degrees. Then the corresponding matrices $P, s$ and $S$ are as follows.

$$
P=\left[\begin{array}{ccc}
1 & 1 & 2 \\
1 & 1 & -2 \\
1 & -1 & 0
\end{array}\right], \quad s=\left[\begin{array}{ccc}
1 & 1 & \sqrt{2} \\
1 & 1 & -\sqrt{2} \\
1 & -1 & 0
\end{array}\right] \quad \text { and } S=\left[\begin{array}{ccc}
1 / 2 & 1 / 2 & 1 / \sqrt{2} \\
1 / 2 & 1 / 2 & -1 / \sqrt{2} \\
1 / \sqrt{2} & -1 / \sqrt{2} & 0
\end{array}\right]
$$

Proof. Let $\delta\left(b_{i}\right)=k_{i}$, for all $i$. Since the integral degrees of $A$ divide the order, $k_{1}$ divides $1+k_{2}$, and $k_{2}$ divides $1+k_{1}$. Therefore, the only possible degree pattern of $A$ are $[1,1,2]$ and $[1,2,3]$, up to the permutations. Since $N_{012}=0,1-\frac{v}{k_{1}}-\frac{u}{k_{2}}=0$. Therefore, the degree patterns $[1,1,2]$ and $[1,2,3]$ imply $v=1-\frac{u}{2}$ and $v=2-\frac{2 u}{3}$, respectively.

Case 1 . Let the degree pattern be $[1,1,2]$, that is, $k_{1}=1$ and $k_{2}=2$.

Therefore, $v=1-\frac{u}{2}$. Since $N_{011}=1, u^{3}(u-1)=0$. Hence $(u, v)=(0,1)$, or $(u, v)=(1,1 / 2)$.

Subcase 1 . Let $(u, v)=(0,1)$. 
Therefore,

$$
P=\left[\begin{array}{ccc}
1 & 1 & 2 \\
1 & 1 & -2 \\
1 & -1 & 0
\end{array}\right], s=\left[\begin{array}{ccc}
1 & 1 & \sqrt{2} \\
1 & 1 & -\sqrt{2} \\
1 & -1 & 0
\end{array}\right] \text { and } S=\left[\begin{array}{ccc}
1 / 2 & 1 / 2 & 1 / \sqrt{2} \\
1 / 2 & 1 / 2 & -1 / \sqrt{2} \\
1 / \sqrt{2} & -1 / \sqrt{2} & 0
\end{array}\right]
$$

Subcase 2. Let $(u, v)=\left(1, \frac{1}{2}\right)$.

Therefore,

$$
P=\left[\begin{array}{ccc}
1 & 1 & 2 \\
1 & \frac{-3+\sqrt{17}}{4} & \frac{-1-\sqrt{17}}{4} \\
1 & \frac{-3-\sqrt{17}}{4} & \frac{-1+\sqrt{17}}{4}
\end{array}\right]
$$

Note that $\delta\left(b_{1}\right)=\delta\left(b_{0}\right)$, but $d_{1} \neq d_{0}$, a contradiction. Hence $u=1$ and $v=\frac{1}{2}$ is not a possible case.

Case 2. Let the degree pattern be $[1,2,3]$, that is, $k_{1}=2$ and $k_{2}=3$.

Therefore, $v=2-\frac{2 u}{3}$. Since $N_{011}=1,625 u^{4}-1850 u^{3}+2520 u^{2}-1296 u+243=0$. But it has no real roots, see [9]. Thus we rule out $[1,2,3]$ degree pattern, because an $s$-matrix associated with a symmetric $C$-algebra might be a real matrix.

Remark 4.3. The above $P$-matrix is given by the character table of the adjacency algebra of an association scheme as4(2), see [6].

In the next theorem we prove that there is only one asymmetric $C$-algebra of rank 3 arising from a Fourier matrix. Moreover, the following theorem shows that for rank 3 it is not necessary to assume $\left|s_{i j}\right| \leq s_{0 j}$ to prove that the homogeneous $C$-algebra arising from a Fourier matrix is a group algebra, see [11, Theorem 13].

Theorem 4.4. Let $(A, \mathbf{B}, \delta)$ be an asymmetric $C$-algebra arising from a Fourier matrix $S$ of rank 3. Then the P-matrix is the first eigenmatrix of the group algebra of a group of order 3 .

Proof. The $P$-matrix of an asymmetric $C$-algebra of rank 3 is as follows,

$$
P=\left[\begin{array}{ccc}
1 & k & k \\
1 & \alpha & \bar{\alpha} \\
1 & \bar{\alpha} & \alpha
\end{array}\right]
$$

where $\alpha=(-1+i \sqrt{1+2 k}) / 2$. Since $A$ is homogenous, $k=1$, see [11, Proposition 12]. Therefore,

$$
P(=s)=\left[\begin{array}{ccc}
1 & 1 & 1 \\
1 & \zeta_{3} & \zeta_{3}^{2} \\
1 & \zeta_{3}^{2} & \zeta_{3}
\end{array}\right] \text { and } S=\frac{1}{\sqrt{3}}\left[\begin{array}{ccc}
1 & 1 & 1 \\
1 & \zeta_{3} & \zeta_{3}^{2} \\
1 & \zeta_{3}^{2} & \zeta_{3}
\end{array}\right]
$$

\section{Fourier matrices of rank 4}

In this section we classify the Fourier matrices under certain conditions, and we show that there is no non-homogeneous integral Fourier matrix of rank 4. For homogenous Fourier matrices see [11]. 
Lemma 5.1. Let $(A, \mathbf{B}, \delta)$ be a $C$-algebra arising from a Fourier matrix $S$ of rank $r$. Let $\left|s_{i j}\right| \leq s_{0 j}$, for all $j$. Let $\delta\left(b_{j}\right)=k_{j}$ for all $j$, and $k_{i}=1$ for some $i>0$.

1. Then $\left|p_{i j}\right|=k_{j}$ for all $j$.

2. If $s$ is a real matrix then $p_{i j}= \pm k_{j}$ for all $j$.

Proof. (i). Since $\delta\left(b_{i}\right)=1, d_{i}=d_{0}$. Therefore, the row 1 and row $i$ of the $s$-matrix are $\left[1,1, \sqrt{k_{2}}, \ldots\right.$, $\left.\sqrt{k_{r-1}}\right]$ and $\left[1, p_{i 1}, p_{i 2} / \sqrt{k_{2}}, \ldots, p_{i, r-1} / \sqrt{k_{r-1}}\right]$, respectively. Since $d_{0}=d_{i},\left|p_{i j}\right| / \sqrt{k_{j}}=\sqrt{k_{j}}$, for all $j$. Hence $\left|p_{i j}\right|=k_{j}$, for all $j$.

(ii). By Part $(i),\left|p_{i j}\right|=k_{j}$ for all $j$. Since $s$ is a real matrix, $p_{i j}= \pm k_{j}$ for all $j$.

The next proposition classify the non-homogeneous Fourier matrices of rank 4 with one nontrivial degree equal to 1.

Proposition 5.2. Let $(A, \mathbf{B}, \delta)$ be a non-homogeneous rational $C$-algebra arising from a Fourier matrix $S$ of rank 4. Let $\left|s_{i j}\right| \leq s_{0 j}$, for all $j$. Let $\delta\left(b_{j}\right)=k_{j}$, for all $j$, and $k_{i}=1$, for some $i>0$. Then the associated P-matrix is

$$
\left[\begin{array}{cccc}
1 & 1 & 4 & 6 \\
1 & 1 & 4 & -6 \\
1 & 1 & -2 & 0 \\
1 & -1 & 0 & 0
\end{array}\right]
$$

Proof. Since $A$ is a rational $C$-algebra, the degrees of $A$ are integers, see [11, Proposition 5]. Let $\delta\left(b_{i}\right)=k_{i}$, for all $i$. Without loss of generality, suppose $k_{1}=1$. Therefore, we have

$$
P=\left[\begin{array}{cccc}
1 & 1 & k_{2} & k_{3} \\
1 & p_{11} & p_{12} & p_{13} \\
1 & p_{21} & p_{22} & p_{23} \\
1 & p_{31} & p_{32} & p_{33}
\end{array}\right]
$$

Case 1. If $p_{11}, p_{12}$ and $p_{13}$ are not rational integers.

Since $d_{0}=d_{1},\left|p_{11}\right|=1,\left|p_{12}\right|=k_{2}$ and $\left|p_{13}\right|=k_{3}$. Thus $p_{11}, p_{12}$ and $p_{13}$ cannot be irrational real numbers. Therefore, they can be non-real algebraic integers. Since the structure constants are rational numbers, a complex conjugate of an irreducible character of $A$ is an irreducible character of $A$. Without loss of generality, assume that the third irreducible character is a complex conjugate of the second character. Thus $k_{2}=1$. But $A$ is non-homogenous and $d_{0}=d_{i} \delta\left(b_{i}\right)$, therefore, $k_{3}=3$ and $d_{3}=2$. Since $S$ is a symmetric matrix, $\left|p_{31}\right|=\left|p_{32}\right|=1$, thus $d_{3}>3$, a contradiction.

Case 2. If $p_{11}, p_{12}$ and $p_{13}$ are rational integers.

By Lemma 5.1, $p_{11}= \pm 1, p_{12}= \pm k_{2}$ and $p_{13}= \pm k_{3}$. Since the row sum of $P$-matrix is zero, the second row of $P$-matrix is either $\left[1,-1, k_{2},-k_{2}\right]$ or $\left[1,1, k_{2},-\left(k_{2}+2\right)\right]$.

Subcase 1 . Let the second row of $P$ be $\left[1,-1, k_{2},-k_{2}\right]$.

Then, the first row of $P$-matrix is $\left[1,1, k_{2}, k_{2}\right]$. Since $k_{2}$ divides $d_{0}$ and $A$ is non-homogenous, $k_{2}=2$. Thus, by the symmetry of Fourier matrix $S$ and orthogonality of characters, we have

$$
P=\left[\begin{array}{cccc}
1 & 1 & 2 & 2 \\
1 & -1 & 2 & -2 \\
1 & 1 & -1 & -1 \\
1 & -1 & -1 & 1
\end{array}\right]
$$

But the $s$-matrix associated to the above $P$-matrix does not have integral structure constants.

Subcase 2. Let the second row of $P$ be $\left[1,1, k_{2},-\left(k_{2}+2\right)\right]$. 
Then, the first row of $P$-matrix is $\left[1,1, k_{2}, k_{2}+2\right]$. Since $k_{2}$ divides $d_{0}$ and $A$ is non-homogenous, $k_{2}=2$ or 4 . Thus, by the symmetry of Fourier matrix $S$ and orthogonality of characters, we have

$$
P=\left[\begin{array}{cccc}
1 & 1 & 2 & 4 \\
1 & 1 & 2 & -4 \\
1 & 1 & -2 & 0 \\
1 & -1 & 0 & 0
\end{array}\right] \text { or } P=\left[\begin{array}{cccc}
1 & 1 & 4 & 6 \\
1 & 1 & 4 & -6 \\
1 & 1 & -2 & 0 \\
1 & -1 & 0 & 0
\end{array}\right]
$$

But the $s$-matrix associated to the first $P$-matrix does not have integral structure constants.

Remark 5.3. The above $P$-matrix is the first eigenmatrix of the adjacency algebra of an association scheme as12(8), see [6].

Cuntz, with a computer, shows that there is no non-homogenous rational Fourier matrix of rank 4, see [3]. In the next theorem, we use $C$-algebra perspective to show that there is no non-homogeneous rational Fourier matrix of rank 4 , that is, there is no non-homogeneous $s$-matrix with integral entries. Unlike the above proposition, we do not assume any nontrivial degree equal to 1.

Theorem 5.4. There is no non-homogenous rational Fourier matrix $S$ of rank 4.

Proof. Let $(A, \mathbf{B}, \delta)$ be a $C$-algebra arising from a rational Fourier matrix $S$ of rank 4 . Let $\delta\left(b_{i}\right)=k_{i}$, for all $i>0$. Since $s$-matrix is integral, $k_{1}, k_{2}$ and $k_{3}$ are square integers, see [11, Proposition 5 (iii)]. As $d_{0}=1+k_{1}+k_{2}+k_{3}$ and $k_{1}, k_{2}$ and $k_{3}$ divide $d_{0}$, therefore, $k_{2}+k_{3} \equiv-1 \bmod k_{1}, k_{1}+k_{3} \equiv-1 \bmod k_{2}$ and $k_{1}+k_{2} \equiv-1 \bmod k_{3}$.

Claim: $k_{1}=k_{2}=k_{3}=1$. Without loss of generality, suppose $k_{1}$ is an even integer. Since $k_{1}, k_{2}$ and $k_{3}$ are squares, $k_{1} \equiv 0 \bmod 4$ and $d_{0} \not \equiv 0 \bmod 4$, a contradiction to the fact that $k_{1}$ divides $d_{0}$. Therefore $k_{1}, k_{2}$ and $k_{3}$ are odd integer. Suppose $k_{1} \geq k_{2}, k_{3}$ and $k_{1}>1$. Now, if all $k_{1}, k_{2}$ and $k_{3}$ are odd integers then $k_{1}, k_{2}, k_{3} \equiv 1 \bmod 4$. But $d_{0} \equiv 0 \bmod 4$ implies $d_{0}=k_{1} a, a \geq 4$. Therefore, $k_{1}(a-1)=1+k_{2}+k_{3}$ implies $3 k_{1} \leq 1+k_{2}+k_{3}$. Thus $k_{2}$ or $k_{3}>k_{1}$, again a contradiction.

\section{Fourier matrices of rank 5}

In this section we prove that there is no non-homogenous $s$-matrix with integral entries (integral Fourier matrix) of rank 5 . But the following proposition shows, under certain conditions, that there are three $s$-matrices of rank 5 with algebraic integer entries. Recall that, a Fourier matrix $S$ with rational entries has associated integral $s$-matrix, and a complex Fourier matrix $S$ has associated $s$-matrix with algebraic integer entries.

Proposition 6.1. Let $(A, \mathbf{B}, \delta)$ be a non-homogeneous rational $C$-algebra arising from a Fourier matrix of rank 5. If $\left|s_{i j}\right| \leq s_{0 j}$ for all $j$. If $\delta\left(b_{i}\right)=1$ for one $i>0$ and $\delta\left(b_{j}\right)=k_{j}$ for all $j \neq i$. Then up to simultaneous row and column permutations the $P$-matrices are as follows,

$$
\left[\begin{array}{ccccc}
1 & 1 & 2 & 2 & 2 \\
1 & 1 & 2 & -2 & -2 \\
1 & 1 & -2 & 0 & 0 \\
1 & -1 & 0 & \sqrt{2} & -\sqrt{2} \\
1 & -1 & 0 & -\sqrt{2} & \sqrt{2}
\end{array}\right], \quad\left[\begin{array}{ccccc}
1 & 1 & 2 & 4 & 8 \\
1 & 1 & 2 & 4 & -8 \\
1 & 1 & 2 & -4 & 0 \\
1 & 1 & -2 & 0 & 0 \\
1 & -1 & 0 & 0 & 0
\end{array}\right] \text { and }\left[\begin{array}{ccccc}
1 & 1 & 4 & 3 & 3 \\
1 & 1 & 4 & -3 & -3 \\
1 & 1 & -2 & 0 & 0 \\
1 & -1 & 0 & \sqrt{3} & -\sqrt{3} \\
1 & -1 & 0 & -\sqrt{3} & \sqrt{3}
\end{array}\right] \text {. }
$$

Proof. Since $A$ is a rational $C$-algebra, the degrees of $A$ are integers. Let $\delta\left(b_{i}\right)=k_{i}$, for all $i$. Without loss of generality, let $k_{1}=1$. Therefore, first eigenmatrix of $A$ is given by

$$
P=\left[\begin{array}{ccccc}
1 & 1 & k_{2} & k_{3} & k_{4} \\
1 & p_{11} & p_{12} & p_{13} & p_{14} \\
1 & p_{21} & p_{22} & p_{23} & -\left(1+p_{21}+p_{22}+p_{23}\right) \\
1 & p_{31} & p_{32} & p_{33} & -\left(1+p_{31}+p_{32}+p_{33}\right) \\
1 & p_{41} & p_{42} & p_{43} & -\left(1+p_{41}+p_{42}+p_{43}\right)
\end{array}\right]
$$


Case 1. If $p_{11}, p_{12}, p_{13}$ and $p_{14}$ are not all rational integers.

Since $d_{0}=d_{1},\left|p_{11}\right|=1,\left|p_{12}\right|=k_{2},\left|p_{13}\right|=k_{3}$ and $\left|p_{14}\right|=k_{4}$. Since the row sum is zero, at least two of these $p_{11}, p_{12}, p_{13}$ and $p_{14}$ can be non-real algebraic integers. Since the structure constants are rational numbers, a complex conjugate of an irreducible character is an irreducible character. Without loss of generality, we assume that the third irreducible character is a complex conjugate of the second character, thus $k_{2}=1$. Without loss of generality, let $k_{3} \leq k_{4}$. Therefore, $d_{0}=d_{i} m_{i}$ implies $\left(k_{3}, k_{4}\right) \in$ $\{(1,2),(1,4),(2,5),(3,6),(6,9)\}$.

If $k_{3}=1$ then $d_{3}=d_{0}=d_{1}=d_{2}$ and $\left|s_{i j}\right| \leq s_{0 j}$ imply all the entries of the rows $1,2,3$ and 4 are nonzero. Since $k_{3} \neq k_{4}$, the entries of the fifth row are rational integers because Galois conjugate of an irreducible character is an irreducible character, and rows of $s$-matrix corresponding to the conjugate characters have equal norm. But $k_{3}=1$ implies $d_{4} \leq 3$. Thus there might be at least two zero entries in the fifth row of $P$-matrix. But $S$ is a symmetric matrix, we get a contradiction.

For $\left(k_{3}, k_{4}\right) \in\{(2,5),(3,6),(6,9)\}, k_{3} \neq k_{4}$, thus the entries of the row 5 are rational integers because $k_{2}=k_{1}=k_{0}=1$ and the Galois conjugate of an irreducible character is an irreducible character. Each entry of the row 1,2 and 3 of $P$-matrix is non-zero. But for each of the above pair there are exactly 3 zeros in the fifth row. Since $S$ is a symmetric matrix, we get a contradiction.

Case 2. If $p_{11}, p_{12}, p_{13}$ and $p_{14}$ are all rational integers.

By Lemma 5.1, the only possible degree patterns are:

$$
\begin{aligned}
& {\left[1,-1, k_{2}, k_{3},-\left(k_{2}+k_{3}\right)\right],\left[1,1, k_{2}, k_{3},-\left(k_{2}+k_{3}+2\right)\right],\left[1,1, k_{2},-k_{3},-\left(k_{2}-k_{3}+2\right)\right],} \\
& {\left[1,-1, k_{2},-k_{3},-\left(k_{2}-k_{3}\right)\right],\left[1,1,-k_{2},-k_{3}, k_{2}+k_{3}-2\right],\left[1,-1,-k_{2},-k_{3}, k_{2}+k_{3}\right] .}
\end{aligned}
$$

Subcase 1 . Let the second row of $P$ be $\left[1,-1, k_{2}, k_{3},-\left(k_{2}+k_{3}\right)\right]$.

Then the first row of the character table is $\left[1,1, k_{2}, k_{3}, k_{2}+k_{3}\right]$ and $\left(k_{2}+k_{3}\right) \mid\left(2+k_{2}+k_{3}\right)$. Thus $\left(k_{2}, k_{3}\right)=(1,1)$. Therefore, by the orthogonality of characters, we have

$$
P=\left[\begin{array}{ccccc}
1 & 1 & 1 & 1 & 2 \\
1 & -1 & 1 & 1 & -2 \\
1 & 1 & p_{22} & p_{23} & -1 \\
1 & 1 & p_{32} & p_{33} & -1 \\
1 & -1 & p_{42} & p_{43} & 1
\end{array}\right]
$$

Since $k_{2}=k_{3}=1, d_{2}=d_{3}=6$. But each of $\left|p_{22}\right|,\left|p_{23}\right|,\left|p_{32}\right|$ and $\left|p_{33}\right|$ can be at most 1 . Thus both $d_{2}$ and $d_{3}$ are strictly less than 6 , a contradiction. Hence this case is not possible.

Subcase 2 . Let the second row of $P$ be $\left[1,1, k_{2}, k_{3},-\left(k_{2}+k_{3}+2\right)\right]$.

Then the first row of the character table is $\left[1,1, k_{2}, k_{3}, k_{2}+k_{3}+2\right]$. Therefore, by the orthogonality of the irreducible characters and symmetry of the matrix $S$, we have

$$
P=\left[\begin{array}{ccccc}
1 & 1 & k_{2} & k_{3} & k_{2}+k_{3}+2 \\
1 & 1 & k_{2} & k_{3} & -\left(k_{2}+k_{3}+2\right) \\
1 & 1 & p_{22} & -2-p_{22} & 0 \\
1 & 1 & p_{32} & -2-p_{32} & 0 \\
1 & -1 & 0 & 0 & 0
\end{array}\right]
$$

Without loss of generality, let $k_{2} \leq k_{3}$. Since $k_{2} \mid\left(2 k_{3}+4\right)$ and $k_{3} \mid\left(2 k_{2}+4\right)$, we have

$$
\left(k_{2}, k_{3}\right) \in\{(1,2),(1,3),(1,6),(2,4),(2,8),(3,10),(4,6),(4,12),(6,16),(8,10),(12,28)\} .
$$

Note that $k_{2} \neq k_{3}$, thus $d_{3} \neq d_{4}$. The structure constants are rational. Therefore, if $p_{22}$ or $p_{33}$ is not rational then row 3 and 4 of $P$-matrix should be Galois conjugates. But the rows of $s$-matrix corresponding to conjugate irreducible characters should have equal norm. Thus $p_{22}$ and $p_{32}$ are rational integers. Therefore, $\operatorname{det}(P) \in \mathbb{Z}$ and $(\operatorname{det} P)^{2}=n^{5}$. Thus $n=2\left(k_{2}+k_{3}+2\right)$ need to be a square. But the only two pairs $(2,4),(4,12)$ do not fail this test. For $\left(k_{2}, k_{3}\right)=(2,4), d_{2}=8=1+1+\left(\frac{p_{22}}{\sqrt{2}}\right)^{2}+\left(\frac{-2-p_{22}}{\sqrt{4}}\right)^{2}$. 
Since the entries of $P$-matrix are algebraic integers, we have $p_{22}=2$. Similarly, $d_{3}=4$ implies $p_{32}=-2$. Therefore,

$$
P=\left[\begin{array}{ccccc}
1 & 1 & 2 & 4 & 8 \\
1 & 1 & 2 & 4 & -8 \\
1 & 1 & 2 & -4 & 0 \\
1 & 1 & -2 & 0 & 0 \\
1 & -1 & 0 & 0 & 0
\end{array}\right]
$$

For $\left(k_{2}, k_{3}\right)=(4,12), d_{2}=9$ and $d_{3}=3$. Therefore, we have $p_{22}=4,-5$ and $p_{32}=1,-2$. Since $S$ is a symmetric matrix, $p_{22}=4$ and $p_{32}=-2$ is the only possibility. But for $p_{22}=4$ and $p_{32}=-2$, the associated $s$-matrix does not have integral structure constants.

Subcase 3 . Let the second row of $P$ be $\left[1,1, k_{2},-k_{3},-\left(k_{2}-k_{3}+2\right)\right]$.

Then, the first row of the character table is $\left[1,1, k_{2}, k_{3}, k_{2}-k_{3}+2\right]$. Therefore, by the orthogonality of characters, symmetry of the Fourier matrix $S$ and $P \bar{P}=n I$, we have

$$
P=\left[\begin{array}{ccccc}
1 & 1 & k_{2} & k_{3} & k_{2}-k_{3}+2 \\
1 & 1 & k_{2} & -k_{3} & -\left(k_{2}-k_{3}+2\right) \\
1 & 1 & -2 & 0 & 0 \\
1 & -1 & 0 & p_{33} & -p_{33} \\
1 & -1 & 0 & p_{43} & -p_{43}
\end{array}\right]
$$

Therefore, $k_{2}\left|4, k_{3}\right| 2 k_{2}+4,\left(k_{2}-k_{3}+2\right) \mid\left(k_{2}+k_{3}+2\right)$ and $k_{2}-k_{3}+2>0$. Hence $\left(k_{2}, k_{3}\right) \in$ $\{(1,1),(1,2),(2,2),(4,2),(4,3),(4,4)\}$. Since $\left|s_{i j}\right| \leq s_{0 j},\left(k_{2}, k_{3}\right) \notin\{(1,1),(1,2)\}$. For $\left(k_{2}, k_{3}\right)=(2,2)$, $d_{3}=d_{4}=4$. Thus $p_{33} \bar{p}_{33}=2, p_{43} \bar{p}_{43}=2$. But the integrality of the structure constants of $s$-matrix and orthogonality of characters forces $p_{33}= \pm \sqrt{2}$ and $p_{43}=\mp \sqrt{2}$. Therefore, up to simultaneous permutation of row 4 and row 5 , and column 4 and column 5 , we have

$$
P=\left[\begin{array}{ccccc}
1 & 1 & 2 & 2 & 2 \\
1 & 1 & 2 & -2 & -2 \\
1 & 1 & -2 & 0 & 0 \\
1 & -1 & 0 & \sqrt{2} & -\sqrt{2} \\
1 & -1 & 0 & -\sqrt{2} & \sqrt{2}
\end{array}\right]
$$

For $\left(k_{2}, k_{3}\right)=(4,2), d_{3}=6$. Thus $\left|p_{33}\right|=\frac{4}{\sqrt{3}}>2$, a contradiction. For $\left(k_{2}, k_{3}\right)=(4,3), k_{4}=3, d_{3}=4$ and $d_{4}=4$. Thus $\left|p_{33}\right|=\sqrt{3}$ and $\left|p_{43}\right|=\sqrt{3}$. But the integrality of structure constants and orthogonality of characters forces $p_{33}= \pm \sqrt{3}$ and $p_{43}=\mp \sqrt{3}$. Therefore, up to simultaneous permutation of row 4 and row 5 , and column 4 and column 5 , we have

$$
P=\left[\begin{array}{ccccc}
1 & 1 & 4 & 3 & 3 \\
1 & 1 & 4 & -3 & -3 \\
1 & 1 & -2 & 0 & 0 \\
1 & -1 & 0 & \sqrt{3} & -\sqrt{3} \\
1 & -1 & 0 & -\sqrt{3} & \sqrt{3}
\end{array}\right]
$$

Although the structure constants are not all integers, for example $\lambda_{342}=3 / 2$, but the associated $s$-matrix has integral structure constants.

Subcase 4 . Let the second row of $P$ be $\left[1,-1, k_{2},-k_{3},-\left(k_{2}-k_{3}\right)\right]$.

Then, the first row of the character table is $\left[1,1, k_{2}, k_{3}, k_{2}-k_{3}\right]$. Therefore, by the orthogonality of the characters and symmetry of the matrix $S$, we have

$$
P=\left[\begin{array}{ccccc}
1 & 1 & k_{2} & k_{3} & k_{2}-k_{3} \\
1 & -1 & k_{2} & -k_{3} & -\left(k_{2}-k_{3}\right) \\
1 & 1 & 0 & p_{23} & -\left(2+p_{23}\right) \\
1 & -1 & p_{32} & p_{33} & -\left(p_{32}+p_{33}\right) \\
1 & -1 & p_{42} & p_{43} & -\left(p_{42}+p_{43}\right)
\end{array}\right]
$$


Since $P \bar{P}=n I$, from row 1,2 and column 3 , we get $k_{2}=0$, a contradiction.

Subcase 5. Let the second row of $P$ be $\left[1,1,-k_{2},-k_{3}, k_{2}+k_{3}-2\right]$.

Then the first row of the character table is $\left[1,1, k_{2}, k_{3}, k_{2}+k_{3}-2\right]$. Therefore, by the symmetry of the matrix $S$ and orthogonality of characters, we have

$$
P=\left[\begin{array}{ccccc}
1 & 1 & k_{2} & k_{3} & k_{2}+k_{3}-2 \\
1 & 1 & -k_{2} & -k_{3} & k_{2}+k_{3}-2 \\
1 & -1 & p_{22} & -p_{22} & 0 \\
1 & -1 & p_{32} & -p_{32} & 0 \\
1 & 1 & 0 & 0 & -2
\end{array}\right]
$$

Therefore $k_{2}\left|2 k_{3}, k_{3}\right| 2 k_{2},\left(k_{2}+k_{3}-2\right) \mid\left(k_{2}+k_{3}+2\right)$ and $k_{2}+k_{3}-2>0$. Without loss of generality, let $k_{2} \leq k_{3}$. Hence $\left(k_{2}, k_{3}\right) \in\{(1,2),(2,2)\}$. Since $\left|s_{i j}\right| \leq s_{0 j},\left(k_{2}, k_{3}\right) \neq(1,2)$. For $\left(k_{2}, k_{3}\right)=(2,2)$, $d_{2}=d_{3}=4$. Thus $p_{22} \bar{p}_{22}=2, p_{32} \bar{p}_{32}=2$. But the integrality of structure constants and orthogonality of characters forces $p_{22}= \pm \sqrt{2}$ and $p_{32}=\mp \sqrt{2}$. Therefore, up to simultaneous permutation of rows and columns, we have

$$
P=\left[\begin{array}{ccccc}
1 & 1 & 2 & 2 & 2 \\
1 & 1 & -2 & -2 & 2 \\
1 & -1 & \sqrt{2} & -\sqrt{2} & 0 \\
1 & -1 & -\sqrt{2} & \sqrt{2} & 0 \\
1 & 1 & 0 & 0 & -2
\end{array}\right]
$$

Subcase 6. Let the second row of $P$ be $\left[1,-1,-k_{2},-k_{3}, k_{2}+k_{3}\right]$.

Then the first row of the character table is $\left[1,1, k_{2}, k_{3}, k_{2}+k_{3}\right]$. Therefore, by the symmetry of the matrix $S$ and orthogonality of the characters, we have

$$
P=\left[\begin{array}{ccccc}
1 & 1 & k_{2} & k_{3} & k_{2}+k_{3} \\
1 & -1 & -k_{2} & -k_{3} & k_{2}+k_{3} \\
1 & -1 & p_{22} & p_{23} & -1 \\
1 & -1 & p_{32} & p_{33} & -1 \\
1 & 1 & p_{42} & p_{43} & 0
\end{array}\right]
$$

Similar to the Subcase $1, k_{2}=k_{3}=1$ implies $d_{2}=d_{3}=6$, and we get a contradiction.

Remark 6.2. We note that the associated $s$-matrices to the $P$-matrices in the above proposition are not integral Fourier matrices. The first two matrices are the character tables of as08(10), as16(24), respectively, see [6]. The third matrix is not a first eigenmatrix of an adjacency algebra of any association scheme because the structure constants generated by the columns of $P$-matrix are not all nonnegative integers.

In the next theorem, by using the properties of $C$-algebras, we show that there is no non-homogeneous rational Fourier matrix of rank 5 .

Theorem 6.3. There is no non-homogeneous rational Fourier matrix $S$ of rank 5.

Proof. Let $(A, \mathbf{B}, \delta)$ be a non-homogenous $C$-algebra arising from a rational Fourier matrix $S$ of rank 5 . Let $\delta\left(b_{i}\right)=k_{i}$, for all $i$. Since $s$-matrix is integral, $k_{i}$ are perfect square integers, see [11, Proposition 5]. By [3, Lemma 3.7], $d_{0}$ is a square. Therefore, $d_{0} \equiv 0,1 \bmod 4$. Let $d_{0}=k_{4} a, d_{0}=k_{3} b, d_{0}=k_{2} c, d_{0}=k_{1} d$. Then each of $a, b, c$ and $d$ is greater than 1 and a square integer because $A$ is non-homogeneous.

Case 1. If each of $k_{1}, k_{2}, k_{3}, k_{4}$ is an odd integer, then $d_{0}$ is odd. Also, the fact that $k_{1}, k_{2}, k_{3}, k_{4}$ are odd implies $a, b, c, d$ are odd and greater than or equal to 9 . Without loss of generality, let $k_{4} \geq k_{1}, k_{2}, k_{3}$. Therefore, $d_{0} \geq 9 k_{4}, d_{0}=1+k_{1}+k_{2}+k_{3}+k_{4} \leq 1+4 k_{4}$, a contradiction. 
Case 2. If three of $k_{1}, k_{2}, k_{3}, k_{4}$ are odd and one is even, then $d_{0}$ is even. Without loss of generality, suppose $k_{4}$ is even. Thus $d_{0}=k_{4} a$ implies $a \geq 4$.

Subcase 1. If $k_{4}>k_{1}, k_{2}, k_{3}$, then $d_{0} \geq 4 k_{4}, d_{0} \leq 1+\left(k_{4}-1\right)+\left(k_{4}-1\right)+\left(k_{4}-1\right)+k_{4}=4 k_{4}-2$, a contradiction.

Subcase 2. If $k_{4}<$ one of $k_{1}, k_{2}, k_{3}$, say $k_{3}$, so $k_{1}, k_{2} \leq k_{3}$, then $d_{0} \geq 4 k_{3}$ because $b$ is an even square. Thus $d_{0} \leq 1+k_{3}+k_{3}+k_{3}+\left(k_{3}-1\right)=4 k_{3}$ implies $k_{1}=k_{2}=k_{3}$ and $k_{4}=k_{3}-1, d_{0}=4 k_{3}$. Now $d_{0}=4 x^{2}$ because $d_{0}$ is a square and an even integer. Hence $k_{1}=k_{2}=k_{3}=x^{2}$ and $k_{4}=x^{2}-1$. Since $x^{2}-1$ divides $4 x^{2}$ and $x$ is an odd integer, $x^{2}-1$ divides 4 , we get a contradiction.

Case 3. If two of $k_{1}, k_{2}, k_{3}, k_{4}$ are odd and two are even, then $d_{0} \equiv 3 \bmod 4$, a contradiction.

Case 4 . If one of $k_{1}, k_{2}, k_{3}, k_{4}$ is odd and three are even, then $d_{0} \equiv 2 \bmod 4$, a contradiction.

Acknowledgment: The author would like to thank Professor Allen Herman whose valuable suggestions helped him to improve this paper.

\section{References}

[1] Z. Arad, E. Fisman, M. Muzychuk, Generalized table algebras, Israel J. Math. 114(1) (1999) 29-60.

[2] H. I. Blau, Table algebras, European J. Combin. 30(6) (2009) 1426-1455.

[3] M. Cuntz, Integral modular data and congruences, J. Algebraic Combin. 29(3) (2009) 357-387.

[4] P. Francesco, P. Mathieu, D. Sénéchal, Conformal Field Theory, Springer-Verlag, New York, 1997.

[5] T. Gannon, Modular data: The algebraic combinatorics of conformal field theory, J. Algebraic Combin. 22(2) (2005) 211-250.

[6] A. Hanaki, I. Miyamoto, Classification of association schemes with small vertices, 2017, available at: math.shinshu-u.ac.jp/ hanaki/as/.

[7] D. G. Higman, Coherent algebras, Linear Algebra Appl. 93 (1987) 209-239.

[8] J. D. Qualls, Lectures on Conformal Field Theory, arXiv:1511.04074 [hep-th].

[9] E. L. Rees, Graphical Discussion of the Roots of a Quartic Equation, Amer. Math. Monthly 29(2) (1922) 51-55.

[10] M. Schottenloher, A Mathematical Introduction to Conformal Field Theory, Springer-Verlag, Berlin, Heidelberg, 2nd edition, 2008.

[11] G. Singh, Classification of homogeneous Fourier matrices, arXiv:1610.05353 [math.RA].

[12] B. Xu, Characters of table algebras and applications to association schemes, J. Combin. Theory Ser. A $115(8)$ (2008) 1358-1373.

[13] A. Zahabi, Applications of Conformal Field Theory and String Theory in Statistical Systems, Ph.D. dissertation, University of Helsinki, Helsinki, Finland, 2013. 